\title{
CHITOSAN/GELATIN NETWORK BASED BIOMATERIALS IN TISSUE ENGINEERING
}

\author{
Kangde Yao, Jinshu Mao, Yuji Yin, Wenguang liu, Yuanlu Cui, Kalyong Cai, Feng Zhao \\ Research Institute of Polymeric Materials, Tianjin University, Tianjin, China
}

\begin{abstract}
Tissue can be looked upon as a cell composite, where cell expresses its functions, while extra
cellular matrixes (ECMs) secreted provide cell information and do its matrix functions. Here ECMs
are. physical and chemical networks consisted of proteins (e.g. collagen, fibronectin. laminin and vi-
tronectin etc.) and glycosaminoglycans (GAGs, e.g. hyaluronic acid, chodroitin 4 -sulfate, chodroitin
6-sulfate etc.). The idea cell-carrier should be the one, which most mimics the ECM. Therefore, chito-
san in which the N-acetylglucosamine moiety is a structural feature also found in the GAGs, and
gelatin, the partially denatured derivative of collagen were used to develop biomaterials. They will
exhibit related bioactivities, as their analogue and precursor, respectively.
(hitosan gelatin network based biomaterials can be applied as membrane, scaffold, surface
modifier and non-viral vector for DNA delivery in tissue engineering. Some of our activities were re-
ported. These biomaterials have promising perspectives.
Biomed Eng Appl Eng Comm, 2002 (June); 14 : 115 -121.
Keywords: Chitosan, Gelatin, Network, Scaffold, Surface modification, Non-viral DNA release
\end{abstract}
\section{INTRODUCTION}

Chitosan, a 1,4 linked 2-amino-2-deoxy- $\beta$-Dglucan is the deacetylated form of chitin, poly- $\beta-(1-4)$ $\mathrm{N}$-acetyl-D-glucosamine), derived from the shells of cructaceans and exoskeletons of insects. The biopolymer comprised of glucosamine and $\mathrm{N}$-acetylglucosamine. The latter is the moiety of glycosaminoglycans (GAGs). So the biologically stemmed aminopolysaccharide exhibits multiple bioactivities, e.g. low toxicity biocompatibility, biodegradable, antimicrobial and wound-healing properties. Moreover, chitosan elicit minimal foreign body reaction. Chitosan favors both soft and hard tissue regeneration.

Gelatin is obtained through a controlled denature of the fibrous insoluble protein-collagen, which is the major component of skin, bone, and connective tissue. It is characterized of no antigenicity in comparison to

Received: Jan. 4, 2002; Accepted: March 25, 2002 Correspondence: Kangde Yao, Professor, Director Research Institute of Polymeric Materials, Tianjin University, Tianjin 300072, China

E-mail: ripm@public.tjuc.com.cn its precursor. Gelatin is frequently used as matrixes for tissue.

With hopes of increased cytocompatibility and elucidation of a desirable cellular response, chitosan/gelatin based biomaterials have been developed [1]

\section{CHITOSAN/GELATIN NETWORK}

\subsection{Chitosan/gelatin Polyelectrolyte Complex (PEC) Hydrogel Membrane}

A chitosan/gelatin PEC hydrogel membrane was prepared via their solution casting [2]. The electrostatic interaction and hydrogen bonding between the aminopolysaccharide and gelatin (isoelectric point 4.9) enhance both tensile strength (Fig 1) and elongation in swollen state (Fig. 2).

Human keratinocyte growth was evaluated by the MTT formazan assay. Fig 3 showed the cell viability $\left(\mathrm{A}_{490}\right)$. Data implied that the chitosan/gelatin PEC improved cell proliferation performance via addition gelatin. The protein polysaccharide pair which likes glycoprotein in biomimetic sense promotes cell viable ability. 


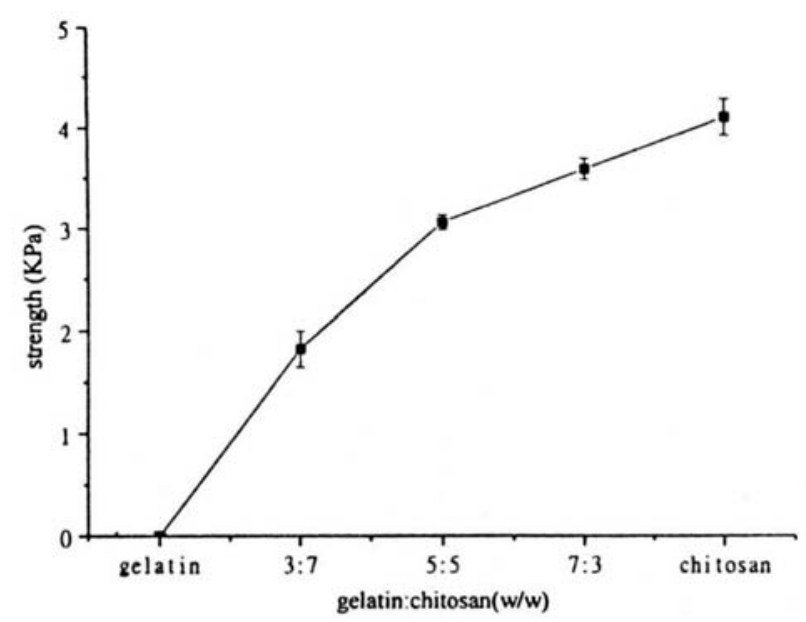

Fig.1 Tensile strength of chitosan-gelatin membranes with different chitosan content, in the swollen state.

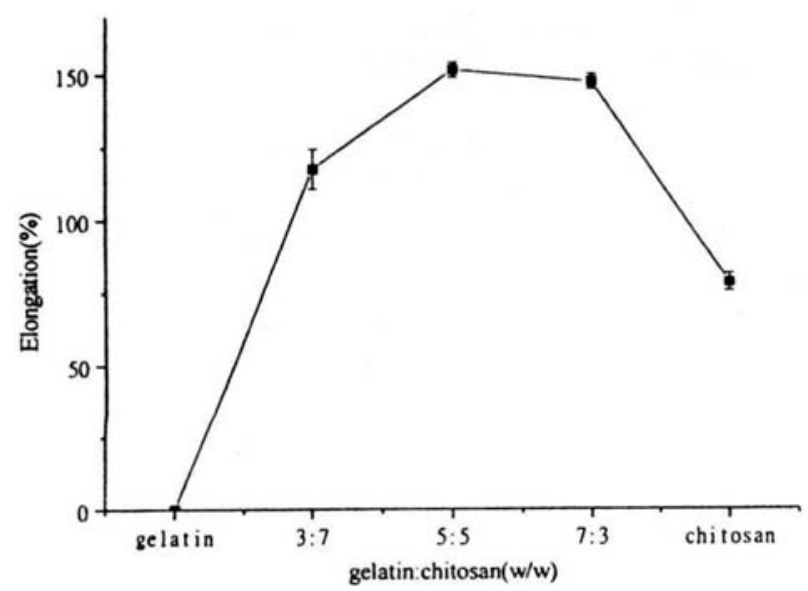

Fig.2 Elongation of chitosan-gelatin membranes with different chitosan content in swollen state.

\subsection{Chitosan/gelatin Network Scaffold (CS- Gel)}

A porous chitosan/gelatin scaffold was fabricated through four main procedures including chitosan/gelatin polyelectrolyte complex hydrogel (CS-Gel PEC) formation, swelling, lyophilization with water in the network as a porogen and crosslinking with glutaraldehyde aqueous solution, while residual aldehyde groups and crosslinking bonding were blocked and reduced with sodium borohydride aqueous solution respectively[3]. The porous matrix obtained was lyophilized again to turn into a chitosan/gelatin network scaffold (CS-Gel). Its porosity depends mainly on the concentration of the original chitosan acetate and gelatin aqueous solution. Moreover, pore size and character relates to the heat transfer performance cf. (Fig 4).

An asymmetric chitosan/gelatin scaffold (CG) was prepared via controlling both the temperature dif-

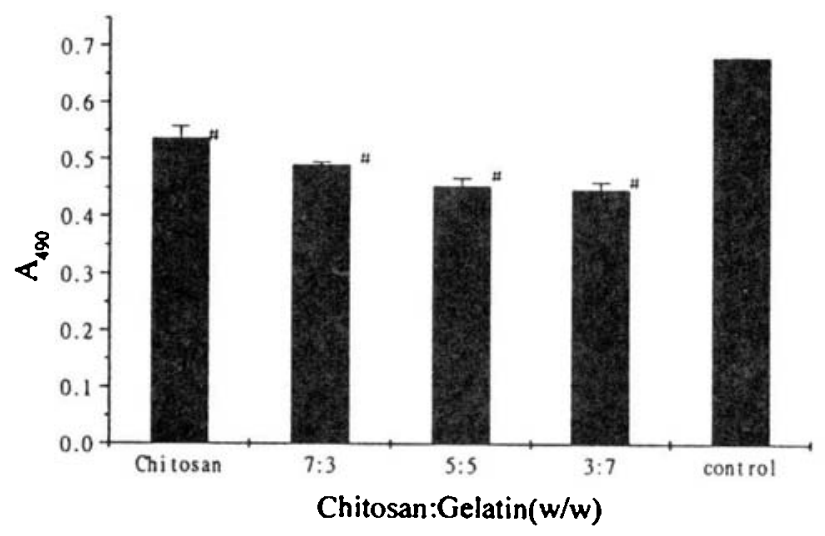

Fig.3 Adhesion of human keratinocytes on chitosangelatin membranes with different chitosan content. $(n=3) . " p<0.05$ relative to chitosan membranes

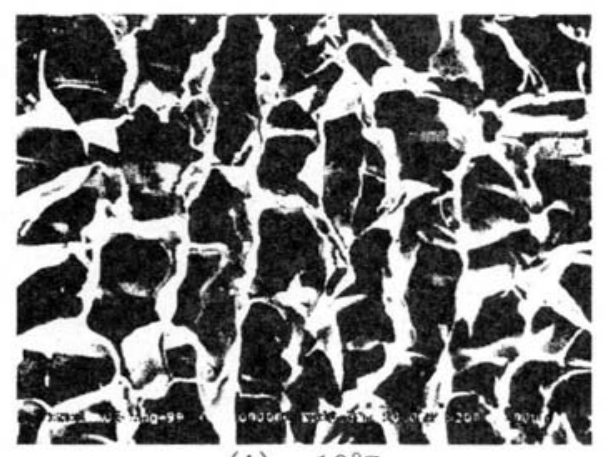

(A) $-10^{\circ} \mathrm{C}$

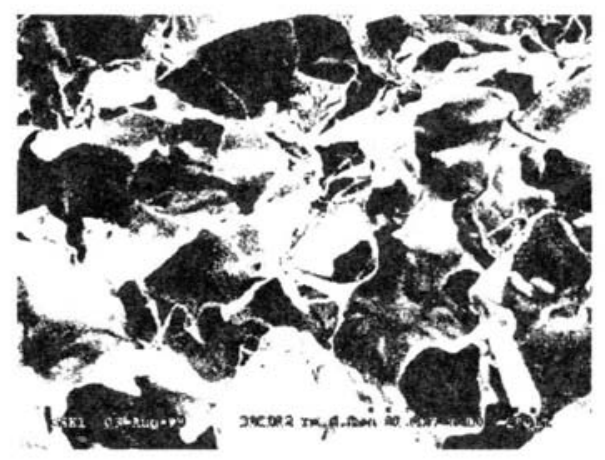

(B) $-40^{\circ} \mathrm{C}$

Fig. 4 SEM micrographs of Cs-Gel scaffolds at different freezing temperature.

ference and heat transfer rate. Fig 5 displays the bilayer CG scaffold with variance in the mean pore size from $30 \mu \mathrm{m}$ to $250 \mu \mathrm{m}$. The CG scaffold was used as a temple for co-cultivation for fibroblast 3 weeks in loose layer and keratinocytes in dense layer 1 week. The fibroblasts/scaffold construct with synthesized extracellular matrices (ECMs) provides suitable microenvironment for keratinocytes growthing and differentiating (Fig. 6). The construct will be a promising skin substitute[4]. 


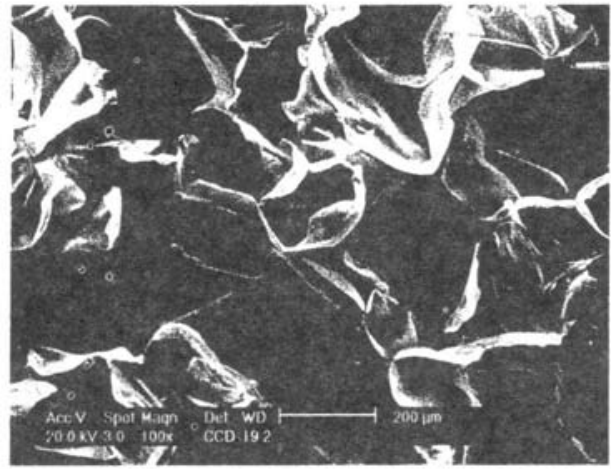

Loose region $(250 \mu \mathrm{m})$

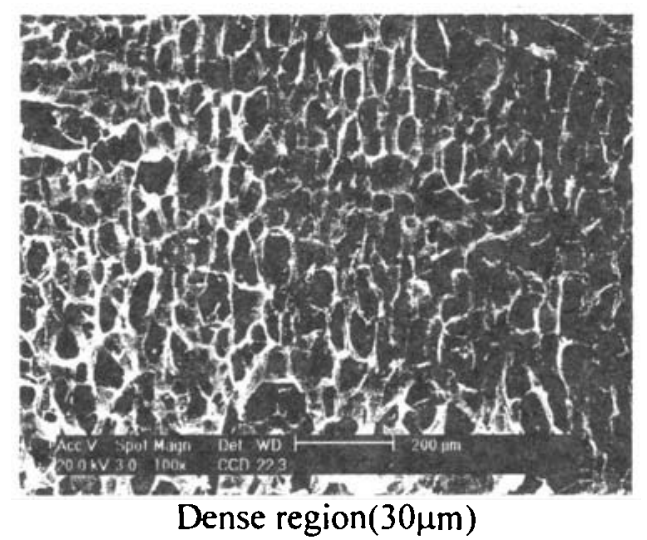

Fig. 5 SEM micrographs of bilayer Cs-Gel-HA scaffolds.

\subsection{Chitosan-gelatin-hyaluronic Acid Scaffold (CS-Gel-HA)}

Hyaluronic acid (HA) as a crucial component of GAGs can promote cell adhesion, growth and migration. It benefits angiogenesis as well. So a CS-Gel-HA scaffold (CHS) was fabricated. Data list in Tab 2 indicated that the presence of HA enhanced its water retention ability and elongation. It is worthy to note that CHS scaffold implanted subcutaneously degradated at $4^{\text {th }}$ week in vivo.

\section{SURFACE MODIFICATION OF POLY $(\alpha$-HYDROXY ACID) WITH CHITOSAN}

Synthetic polymers play an important role in the design and engineered new tissue. They have been obvious candidates for such applications because their chemical and physical properties can be controlled. However, these materials e.g. poly ( $\alpha$-hydroxy acid)s often lack the biosignals in cellular behavior including adhesion, migration, differentiation and proliferation. Although there is no modifying site, the $\operatorname{poly}(\alpha-$

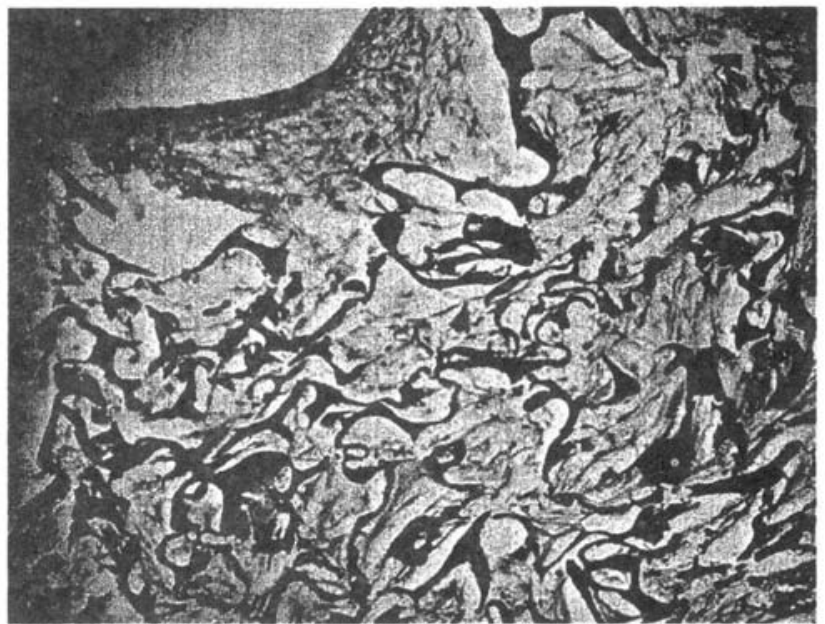

Fig. 6 Keratinocyte (upper layer) co-cultured with fibroblasts (bottom layer) in the scaffolds for one week stained with HE. (fibroblast have been cultured for three week, $\mathrm{HE}$ )

hydroxy acid)s e.g. poly(D,L-lactic acid) (PDLLA). So surface modification of PDLLA membrane was carried out through activation with controlled hydrolysis to set carboxylic groups to PDLLA molecules on the surface and then coupling chitosan molecules with the carboxy-terminated with $\mathrm{N}, \mathrm{N}$-dimethylaminopropyl-Nethylcarbodimide (EDC) in 2-(N-morhpolino)-ethane sulfonic acid (MES) buffer. PDLLA membrane immobilize chitosan improves its hydrophilicity and reduces the contact angle against water to $49 \pm 1^{\circ}$, instead of 69 $\pm 3^{\circ}$ for the PDLLA membrane.

There are significant differences in rat calvaria osteoblast performance, including cell attachment, proliferation kinetics and alkaline phosphatase activity (ALP) on chitosan modified PDLLA membrane (Fig79)[5]. It is very interesting to try to compare the positive result for osteoblasts with that obtained in vivo. Here a porous PDLLA scaffold was modified with chitosan via entrapment approach. The scaffold was treated with a dioxane-water mixture containing chitosan acetate. A gel was formed on its interface layer. The chitosan molecules were entrapped in the surface along with interface collapse by water treatment. Fig 10 displayed a SEM micrograph of chitosan modified PDLLA scaffold. The scaffold was applied to fill $10 \mathrm{~mm}$ defects in rabbit radial bone for 12 weeks. The preliminary animal test result indicated that there is faint foreign body reaction while the new forming bone can be seen infiltrating the defects. The surface zone of PDLLA scaffold was surround with fibrous tissue. Here, the modifier chitosan has a $\mathrm{N}$-acetyl group just like GAGs. The groups pay an essential role in cell function control in vivo. Moreover, the presence of the aminopolysaccharide compensates the $\mathrm{pH}$ pulse effect stemming from degradable product, lactic acid that trigger foreign body inflammatory reaction. Both 
Tab2 Mechanical properties comparison of CG and CGH scaffolds

\begin{tabular}{ccccc}
\hline scaffold & Pore size $(\mu \mathrm{m})$ & Water content $(\mathrm{g} / \mathrm{g})$ & Strenth(Mpa) & Elongation(\%) \\
\hline CG & $128.5 \pm 2.8$ & $20.52 \pm 0.37$ & $0.42 \pm 0.05$ & $46.60 \pm 4.86$ \\
CGH & $140.6 \pm 4.5$ & $25.41 \pm 0.7$ & $0.28 \pm 0.03$ & $68.98 \pm 9.23$ \\
P value & $<0.01$ & $<0.005$ & $<0.005$ & $<0.01$ \\
\hline
\end{tabular}

of these will benefit cytocompatibility for the chitosan modified PDLLA scaffold.

\section{SURFACE MODIFICATION OF POLY $(\alpha$-HYDROXY ACID) WITH GELATIN}

We also investigated the efficiency of two treatments for poly (L-lactic acid) (PLLA) surface modification with gelatin, via entrapment and coupling by using 1-ethyl-3-(3-dimethylaminopropyl)carbodiimide (EDC) and N-hydroxysuccinimide (NHS). The properties of control PLLA films, entrapment and coupled PLLA films were investigated by water contact angle measurement and electron spectroscopy for chemical analysis (ESCA). The contact angle against water indicated the change in hydrophilicity and the ESCA data suggested that the modified PLLA films became ones enriched with nitrogen atoms. The cytocompatibility of modified PLLA films was improved. There are significant differences in bovine articular chondrocyte performance, including cell attachment, proliferation, and cell function (glycosaminoglycan and collagen type II secretion) on gelatin modified PLLA membrane

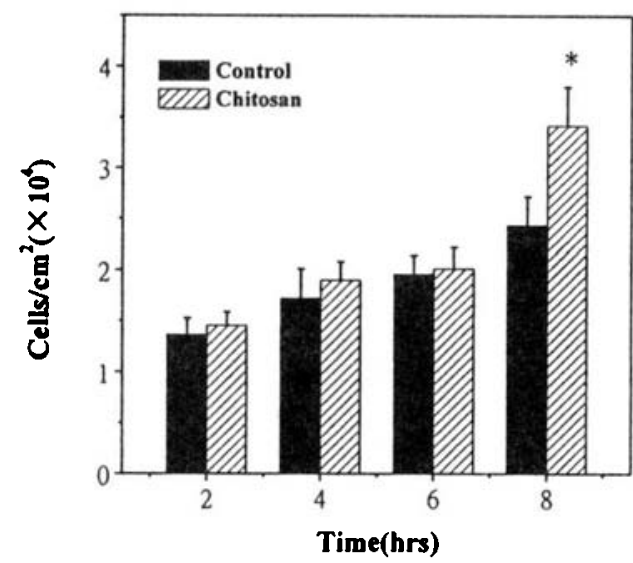

Fig. 7 The attachment of osteoblasts to modified PDLLA film with chitosan and control, initial seeding density was 30000 cells $/ \mathrm{cm}^{2},{ }^{*} \mathbf{P}<0.05$ (compared to control)
(Figl1-14).

\section{HYDROXYAPITATE/CHITOSAN- GELATIN NETWORK SCAFFOLD (HAVCS-GEL)}

Bone is a biocomposite comprised of nanohydroxyapatite (HA) crystalline orienting along collagen fibers mainly. A HA/CS-Gel network composite scaffold was fabricated with freeze-dried method. The SEM micrographs of its fracture surface of the scaffold was shown in Fig 15. The rat calvaria osteoblasts attached and proliferated well within the scaffold. Fig 16 shows the cross section of osteoblasts/scaffold construct for 21 days. The cells proliferated along the pore walls laid down ECMs, which was mainly collagen $\mathrm{I}$.

\section{CONSTITUTION OF GENE AC- TIVATED MODIFIED LAYER}

Combination of chitosan based matrix with gene therapy and the use of expressed growth factors will improve the lone-term outcome of tissue repair in

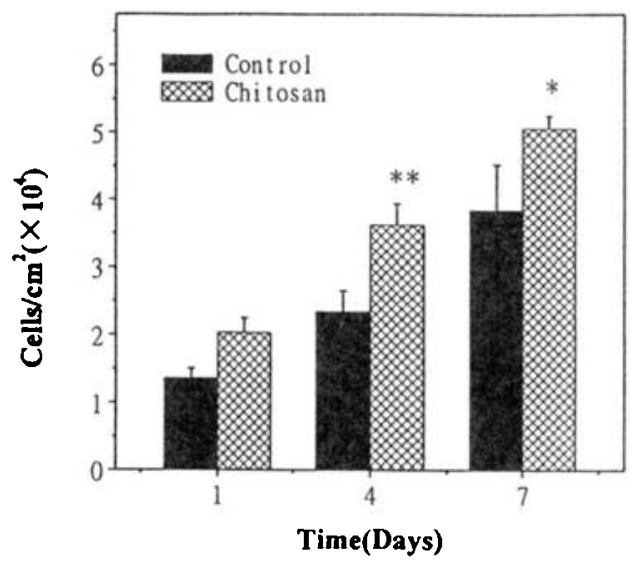

Fig 8 Proliferation kinetics of rat calvaria osteoblasts culture on chitosan-modified PDLLA film and control. Seeding density of 30000 cells $/ \mathrm{cm}^{2}$. Error bars represent means $\pm S D$ for $n=3$. ${ }^{\star} P<0.05$ **P<0.01(compared to control) 


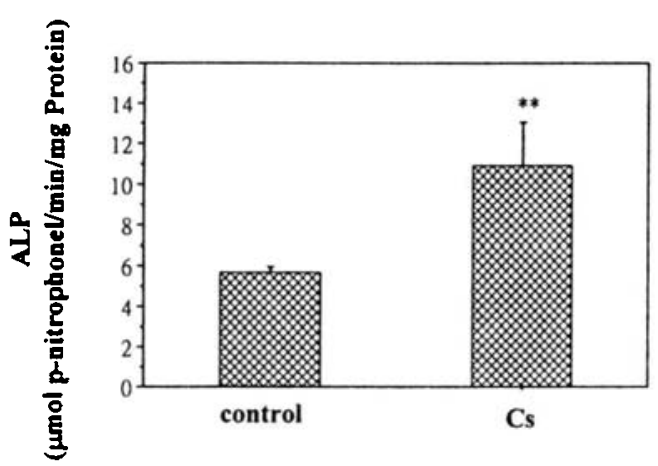

Fig. 9 Alkaline phosphatase activity of osteoblasts seeded on chitosan-modified PDLLA film and control for 7 days. Error bars represent means \pm SD for $n=3$. ( Initial seeding density was $\mathbf{3 0 0 0 0}$ cells $/ \mathrm{cm}^{2}$.). ${ }^{\star \star} p<0.01$ (compared to control).

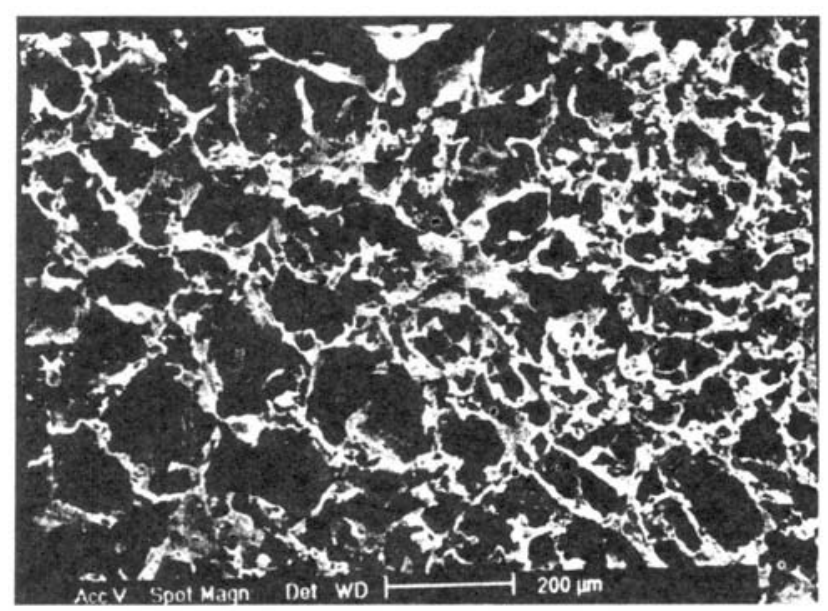

Fig. 10 SEM image of Chitosan modified PDLLA scaffold.

clinical settings. DNA complexation with chitosan has been shown to protect plasmids from degradation by nucleases and also facilitates cellular transfection by poorly understood interaction with cell membrane. With this in mind, dodecylated chitosan(CS-12) was used a non-viral vector for delivery DNA. A DNA/CS12 polyelectrolyte complex (PEC) was prepared. AFM image showed that the PEC developed a globule-like structure composed of 40-115 DNA molecules (Fig 17). DNA can be released via exchange with cations e.g. sodium, calcium and magnesium ions. The released DNA concentration relates to the concentrations of the corresponding salts (Fig 18). DNA molecules remain intact and undegraded due to the protection DNAase offered by CS-12 [8].

For explicating the possibility of application of $\mathrm{N}$-alkylated chitosan to modify PDLLA film, N-butyl chitosan and N-cetyl chitosan were immobilized on the PDLLA activated films. There was significant differ-

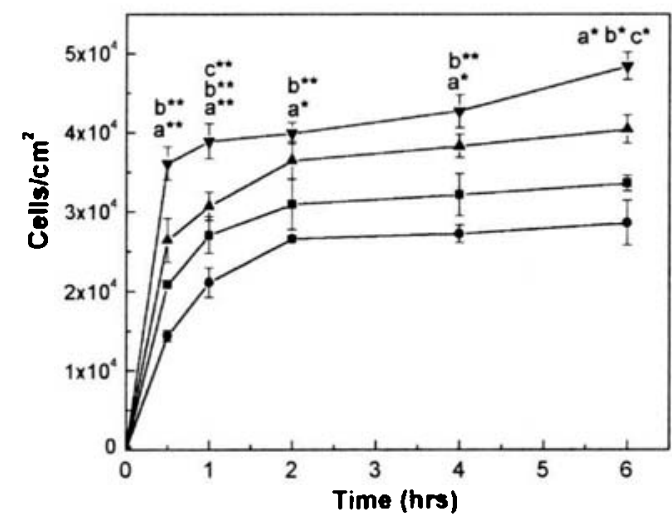

Fig. 11 The attachment of chondrocytes to gelatin modified PLLA films via coupling method $(\nabla)$ and entrapment method $(\Delta)$ as well as control PLLA films (O) and tissue culture plates ( ()$_{\text {) in- }}$ creased over a $6 \mathrm{~h}$ time frame, initial seeding density was $5 \times 10^{4} \mathrm{cells} / \mathrm{cm}^{2}, \quad\left({ }^{\star} P<0.05 ; \quad * \star P<0.01\right.$. a: entrapment compared to control; b: coupling compared to control; and c: entrapment compared to coupling).

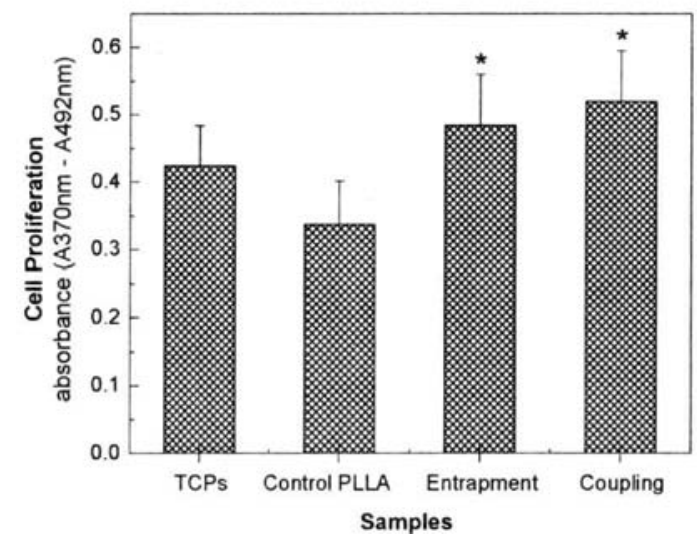

Fig. 12 Measurement of the proliferation of chondrocytes cultured on gelatin modified PLLA film via entrapment and coupling methods as well as control PLLA films and tissue cultures plates (TCPs). After chondrocytes were cultured in 48well plates for $48 \mathrm{~h}$, BrdU was added and cells were reincubated for additional $18 \mathrm{~h}$. BrdU incorporation was determined using a cell ELISA method. (Compared to the control PLLA films, ${ }^{\star} P<0.05$.)

ence for the $\mathrm{N}$-butyl chitosan modified film in comparison to control. (cf. Fig.19)[6]

\section{SUMMARY}




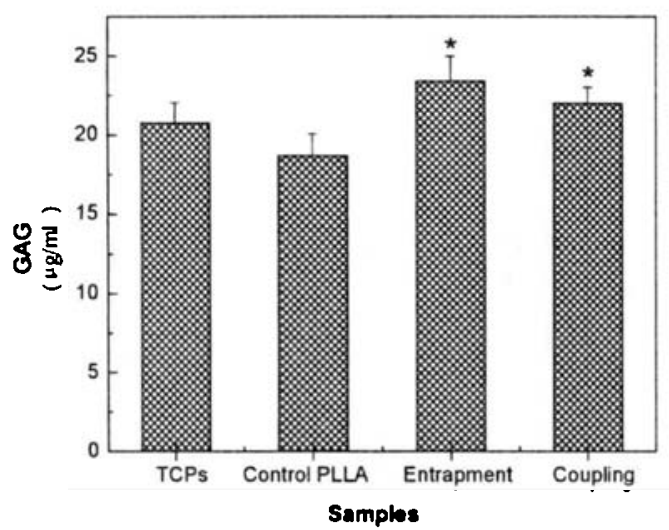

Fig. 13 The total GAG produced by chondrocytes cultured on gelatin modified PLLA film via entrapment and coupling methods as well as control PLLA films and tissue culture plates (TCPs) for 10 days. (Compared to the control PLLA films, $\left.{ }^{\star} \boldsymbol{P}<0.05\right)$

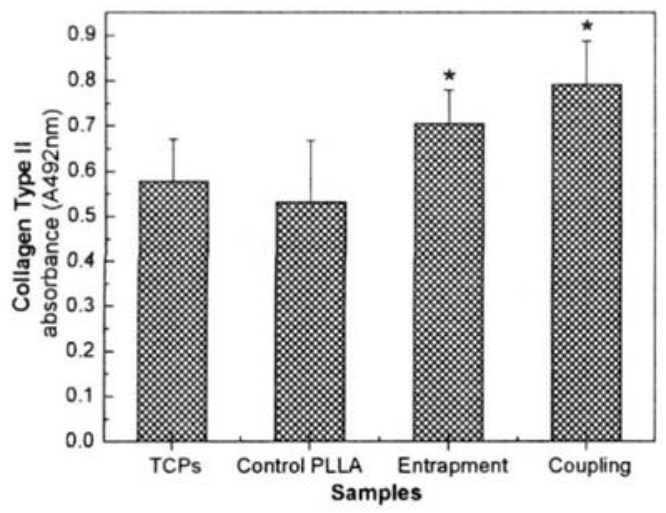

Fig. 14 Collagen Type II secreted by chondrocytes cultured on gelatin modified PLLA film via entrapment and coupling methods as well as control PLLA films and tissue culture plates (TCPs) for 10 days. (Compared to the control PLLA films, $\star P<0.05)$.

A combination of flexible protein and rigid polysaccharide results in a series of biomimetic chitosan/gelatin based biomaterials covering surface modifier and non-viral vector for gene therapy. They held promising perspectives in tissue engineering.

\section{ACKNOWLEDGEMENTS}

The financial support of the main develop project for basic research via G199905305 grant, National

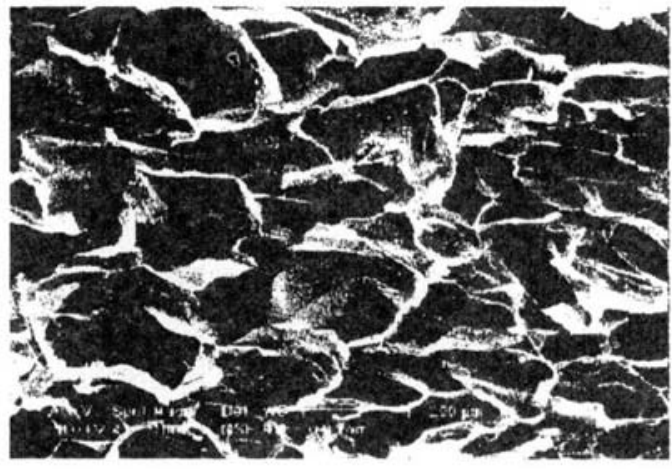

Fig 15. SEM image of HA/CS-Gel scaffold (CSGel\%:2.5\%, HA/CS-Gel:30/70).

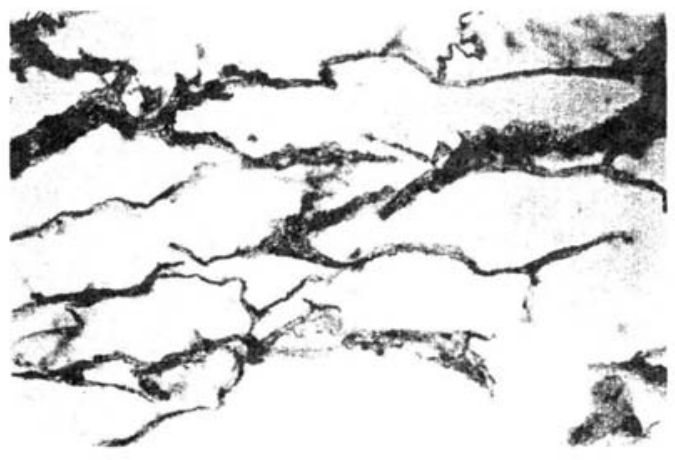

(a)

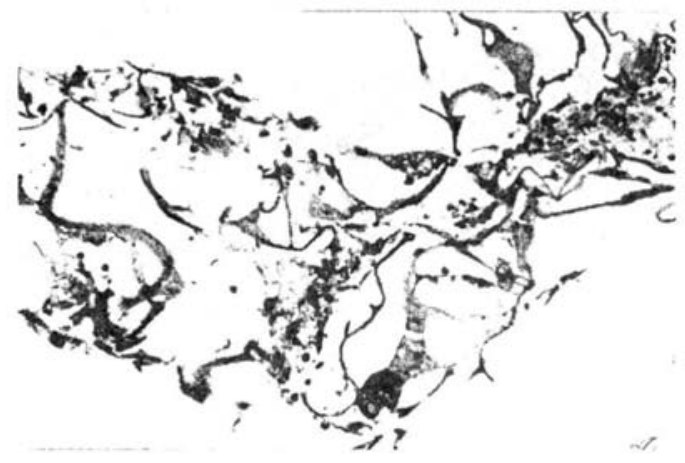

(b)

Fig. 16 Light micrographs of HA/CS-Gel composite scaffold initially seeded with $2.6 \times 10^{6} \mathrm{cells} / \mathrm{cm}^{2}$ and cultured for 21 days. The cross section was stained with type I collagen antibody. Original magnification: $\times 200$. (a) control (b) scaffold/cell construct.

Natural Science Fundation through 59973014 and 5983002 Shanghai Science Fundation via 00DJ140014 are acknowledged. 


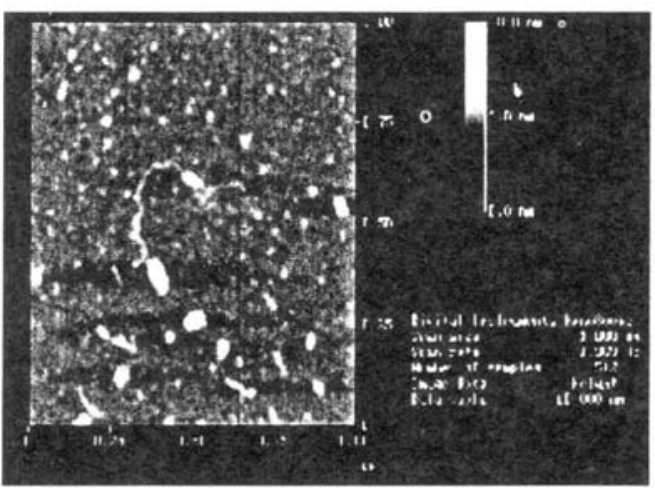

(A)

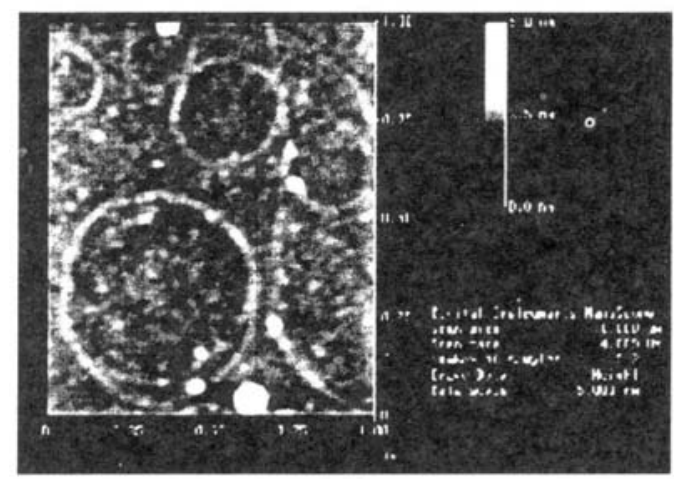

(B)

Fig. 17 AFM images of pure DNA (A) and DNA released from complex (B) with the addition of Dnase.

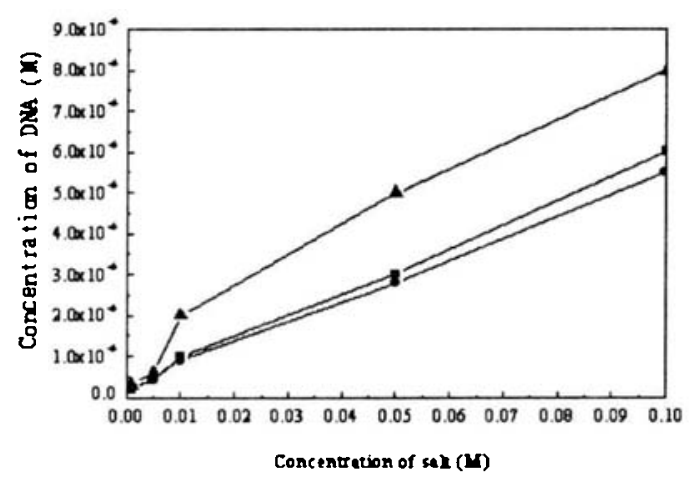

Fig. 18 Dependence of the amount of delivered DNA on the concentration of $\mathrm{NaCl}(\square), \mathrm{KCl}(O)$ and $\mathrm{MgCl}_{2}(\mathbf{A})$ solutions.

\section{REFERENCES}

1. Kang De Yao, Yu Ji Yin, Mei Xuan $X u$ and $Y u$ Fen Wang, Investigation of $\mathrm{pH}$-sensitive drug de-

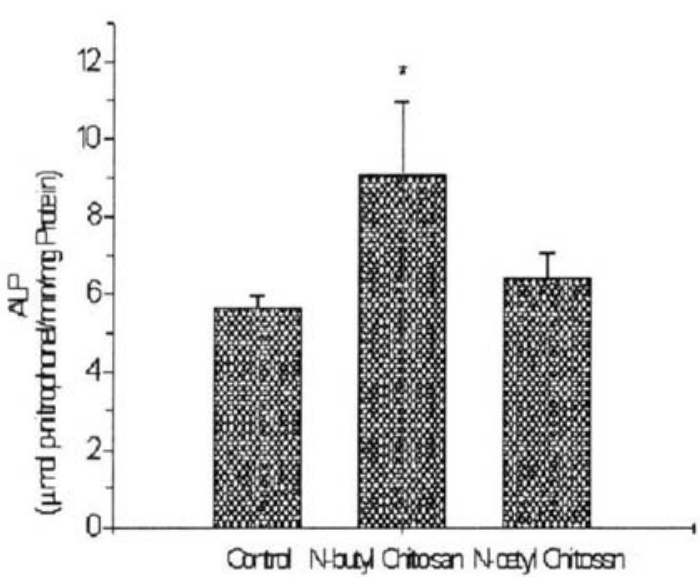

Fig. 19 Alkaline phosphatase activity of osteoblasts seeded on $\mathbf{N}$-alkylated chitosan modified PDLLA film and control for 7 days. Error bars represent means $\pm S D$ for $n=3$. (Initial seeding density was 30000 cells $/ \mathrm{cm}^{2}$.). ${ }^{\star} \mathbf{p}<0.05$ (compared to control).

livery system of chitosan/gelatin hybrid polymer network, Polym. Intern., 1995,38:77-82

2. Y. J. Yin, K. D. Yao, G.X.Cheng and J.B. Ma, Properties of polyelectrolyte complex films of chitosan and gelatin, Polym. Intern., 1999,48:429-432

3. F Shen, Y.L. Cui, L.F. Yang, K.D. Yao, X.H. Hong, W.Y. Jia, H. D. Shi, A study on the fabrication of porous chitosan/gelatin network scaffold for tissue engineering. Polymer Intern., 2000,49: 1596-1599

4. Jinshu Mao,Liguo Zhao, Kang de Yao, Qingxin Shang,Guanghui Yang,Yilin Cao Study on novel chitosan-gelatin artificial skin in vitro. J Biomed. Mater. Res., in press.

5. Kaiyong Cai, Songbai Lin, Kangde Yao, Zhiming Yang, Xioqiong Li, Huiqi Xie, Tingwu Qing, Juan Luo, Surface modification of poly (D,L-lactic acid) with chitosan and its effects on the culture of osteoblast in vitro. J Biomed. Mater. Res., 2002; 60(3): 398-404

6. Kaiyong Cai, Songbai Lin, Kangde Yao, Zhiming Yang, Xioqiong Li, Huiqi Xie, Tingwu Qing, Juan Luo, Modulation of osteoblast function using poly(D,L-lactic acid) surfaces modified with alkylation derivatives of chitosan. J. Biomater Sci, Polym E., 2002,13: 53-66

7. Feng Zhao, Yuji Yin, William W. Lu, J. Chiyan Leong, Wenyi Zhang, Jingyu Zhang, Mingfang Zhang and Kangde Yao, Preparation and histological evaluation of biomimetic three-dimensional hydroxyapatite/ chitosan-gelatin network composite scaffolds. Biomaterials 2002, 23:15:3227-3234

8. Wenguang Liu, Kangde yao, Qinggang Liu, Formation of a DNA/N-dodecylated chitosan complex and salts induced gene delivery. J. Appl. Polum. Sci., 2001,82:3393-3398. 